\title{
Les mobilités dans les aires périurbaines du Grand Tunis. Étude de cas d'El Mornaguia
}

Mobility in the peri-urban areas of Greater Tunis. Case study of El Mornaguia

Souhir Bouzid

\section{(2) OpenEdition}

12 Journals

Édition électronique

URL : https://journals.openedition.org/popvuln/1273

DOI : $10.4000 /$ popvuln.1273

ISSN : 2650-7684

Éditeur

LIR3S - Laboratoire Interdisciplinaire de Recherche "Sociétés Sensibilités Soin" (UMR 7366 CNRS-uB)

Édition imprimée

Date de publication : 1 décembre 2020

Pagination : 65-92

ISBN : 978-2-918173-28-1

ISSN : 2269-0182

Référence électronique

Souhir Bouzid, «Les mobilités dans les aires périurbaines du Grand Tunis. Étude de cas d'El

Mornaguia », Populations vulnérables [En ligne], 6 | 2020, mis en ligne le 01 décembre 2021, consulté le 26 février 2022. URL : http://journals.openedition.org/popvuln/1273 ; DOI : https://doi.org/10.4000/ popvuln. 1273

Ce document a été généré automatiquement le 26 février 2022.

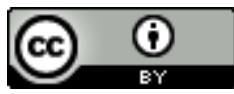

Les contenus de la revue Populations vulnérables sont mis à disposition selon les termes de la Licence Creative Commons Attribution 4.0 International 


\section{Les mobilités dans les aires} périurbaines du Grand Tunis. Étude
de cas d'El Mornaguia

Mobility in the peri-urban areas of Greater Tunis. Case study of El Mornaguia

\section{Souhir Bouzid}

\section{Introduction}

1 La mobilité constitue un atout majeur pour le désenclavement des quartiers périphériques ainsi qu'un enjeu majeur pour la cohésion sociale. Ainsi les conditions des personnes (âge, catégorie socioprofessionnelle, état de santé, situation économique, sexe, etc.) sont en étroite liaison avec les conditions de mobilité. Plus leurs conditions sont meilleures, plus ils se déplacent rapidement et loin. La mobilité est, et deviendra de plus en plus, l'une des conditions de l'intégration sociale et de l'intégration urbaine. En effet, les formes de liens sociaux urbains, la civilisation urbaine, sont basées sur le brassage social, la rencontre de l'autre, du différent.

2 Ainsi, les choix résidentiels des ménages dans les espaces périurbains tunisois diffèrent d'un ménage à un autre, ce qui crée une réelle diversité sociale dans ces espaces. L'étalement spatial important opéré à partir des années 1970 a fait passer l'amplitude de la ville de Tunis du Nord au Sud de $10 \mathrm{~km}$ à $30 \mathrm{~km}$ aujourd'hui, elle atteindra probablement $50 \mathrm{~km}$ dans une quinzaine d'années (Abid et Chabbi, 2008). Cet étalement spatial a sans doute des effets notables sur le transport et la mobilité. De ce fait, les habitants du périurbain ont « des territoires très étendus aux contours flous, variables selon chaque individu » (Pinson et Thomann, 2001).

3 Bourg rural dans les années 1970, El Mornaguia et sa périphérie sont habitées par plus de 40000 habitants actuellement. L'étalement urbain qu'El Mornaguia est en train de connaître se fait aux dépens de ses espaces agricoles. L'installation des ménages y est 
en étroite relation avec le développement des systèmes de transport et de la mobilité des personnes.

4 Nous nous demanderons dans quelle mesure l'installation dans l'aire périurbaine d'El Mornaguia est à l'origine d'inégalités sociales pour une population aux origines diverses.

5 À cet effet, nous chercherons à déterminer dans quelle mesure les conditions sociales et spatiales influencent les mobilités des individus. Nous ne manquerons pas de nous demander comment se vit la mobilité des plus vulnérables, à savoir des jeunes (12-18ans), des aînés (60 ans et plus) et des femmes seules.

6 Cet article analyse les conséquences d'une installation en périurbain à El Mornaguia sur les pratiques de mobilité, à partir d'une enquête par questionnaire auprès de $5 \%$ des ménages et à partir d'entretiens semi-directifs effectués avec des groupes spécifiques en situation de vulnérabilité en matière de mobilité.

\section{Revue de la littérature}

7 Il existe une forte interaction entre mobilité spatiale et mobilité sociale: "les personnes les mieux dotées socialement se déplacent plus rapidement et plus loin " (Kaufmann, 2001). La mobilité constitue un atout majeur pour le désenclavement des quartiers périphériques et aussi un enjeu majeur pour la cohésion sociale. La mobilité est, et deviendra de plus en plus, l'une des conditions de l'intégration sociale et de l'intégration urbaine. En effet, les formes de liens sociaux urbains, la civilisation urbaine, sont basées sur le brassage social, la rencontre de l'autre, du différent. La mobilité est l'une des conditions de la sortie de son quartier, de ses références habituelles, familiales et affectives. La mobilité reflète le mode de vie quotidien. Par ailleurs Kaumann affirme que «La mobilité quotidienne en train, en voiture particulière ou en bus reflète, hier comme aujourd'hui, des inégalités sociales » (Kaufmann 2001). La mobilité participe bel et bien à la différenciation sociale des individus.

8 En outre, l'accès à la mobilité est différencié selon les groupes sociaux et en particulier selon les niveaux de revenus (Orfeuil, 2004). Ainsi, Jouffe, Caubel, Fol et Motte-Baumvol (2015) affirment qu' indépendamment d'un éventuel cumul de handicaps sociaux, la pauvreté en ressources économiques a un effet propre sur la mobilité quotidienne. Ainsi, les ménages pauvres ont des déplacements plus courts et moins fréquents ». Les disparités de mobilité subies par les ménages pauvres peuvent se traduire par des inégalités significatives dans l'accès à des ressources décisives pour les individus, et ce particulièrement pour les ménages localisés dans les territoires moins densément équipés. Ces inégalités de mobilité sont accentuées dans les périphéries des agglomérations urbaines. Ainsi, Mathieu Saujot (2012) affirme que «spatialement, la vulnérabilité en mobilité est plus forte chez les ménages du périurbain, car ce sont eux qui parcourent le plus de kilomètres quotidiens ». La vulnérabilité est définie par le même auteur comme "une situation de tension qui peut aboutir à une situation de précarité, où cette tension a alors des conséquences réelles sur le ménage ».

9 Certains auteurs rejoignent Mathieu Saujot et affirment « que les ménages vulnérables sont globalement situés dans les parties périurbaines des aires urbaines, et sont 
généralement composés de famille des classes moyennes multi-motorisées " (Nicolas, Vanco et Verry, 2012).

Les espaces périurbains ne sont pas homogènes au niveau de l'organisation spatiale ainsi que sociale et les politiques en matière de déplacement diffèrent d'un espace à un autre. Il y a une différence au niveau du périurbain lui-même : périurbain proche et périurbain lointain, périurbain communal, périurbain non communal, ce qui a pour effet aussi d'augmenter ces inégalités. Dans le même ordre d'idées, Orfeuil (2010), en étudiant les différentes formes d'inégalités dans les espaces périurbains, atteste que tout accident, toute rupture dans le cours normal des choses peuvents'avérer problématiques : une suppression de permis ou une grosse réparation imprévue peuvent entraîner une rupture du lien avec 1

'employeur[...],toute fermeture d'entreprise implique une recherche d' emploi sur un espace très étendu, où le coût de la seule migration vers le travail sera mis en balance avec les ressources procurées, et cette balance ne sera pas toujours estimée positive.

Certains chercheurs rejoignent Orfeuil et attestent que les inégalités de mobilité sont plus fortes dans les périphéries urbaines (Jouffe, Caubel, Fol et Motte-Baumvol, 2015). Ces auteurs affirment également que « Les disparités de mobilité subies par les ménages pauvres peuvent se traduire par des inégalités significatives dans l'accès à des ressources décisives pour les individus, et ce particulièrement pour les ménages localisés dans les territoires moins densément équipés ».

Plusieurs études sur la mobilité soulignent que l'accès à l'automobile constitue une condition nécessaire pour une bonne intégration sociale (Orfeuil, 2004). Quelle qu'en soit la raison, économique, physique ou cognitive, ne pas avoir accès à l'automobile est le facteur le plus explicatif des inégalités constatées en termes de mobilité urbaine quotidienne. Par opposition, une fois la motorisation acquise, les inégalités de mobilité apparaissent minimes (Nicolas, Vanco et Verry, 2012). En outre, la possession d'une voiture impose des coûts élevés aux ménages pauvres. Comme la participation au travail dépend de l'automobilité des travailleurs peu qualifiés, les dépenses en voiture sont devenues plus lourdes pour les ménages à faible revenu (Froud et al., 2002). Dans la même optique Coquard (2018) affirme que « l'investissement dans la voiture compense la délocalisation des emplois » et par conséquent facilite l'insertion professionnelle.

En outre, la mobilité des ménages pauvres s'effectue sur un territoire relativement restreint et l'éloignement de l'emploi du domicile est un facteur déterminant dans la recherche d'un emploi. La recherche d'un emploi pour un ménage pauvre se caractérise ainsi par une limitation volontaire du périmètre de la recherche d'emploi et de l'éloignement des emplois susceptibles d'être acceptés, comme l'a montré Fol (2009) à partir de travaux sur des villes en France, en Grande-Bretagne et aux États-Unis.

14 À l'issue de cette section, nous signalons que des conditions sociales et spatiales influencent fortement la mobilité des personnes. Dans la même optique, Kaufmann (2001) résume les inégalités en matière de mobilité en affirmant que "la mobilité quotidienne est un véhicule d'inégalités sociales ». Àcela,Orfeuil (2010) ajoute que

l'aptitude à la mobilité est, bien plus aujourd'hui qu'hier, une condition indispensable à l'insertion, à la construction de trajectoires de vie satisfaisantes et de liens sociaux diversifiés. La mobilité n'est plus seulement un choix, elle est devenue une impérieuse nécessité. Face à cette nécessité, nombreux sont ceux qui sont mal armés, ou moins bien armés que d'autres. Les différences d'aptitude à la 
mobilité font non seulement partie $\mathrm{du}$ "tableau général » des inégalités, mais elles sont aussi partie intégrante de leur reproduction.

\section{Les données}

Nous avons réalisé une enquête sur la mobilité de personnes résidantes à El Mornaguia en 2014. L'échantillon de base était composé de 362 logements et nous nous intéressions aux profils et aux déplacements de tous les membres des ménages de l'échantillon ayant 5 ans ou plus. Les ménages interrogés ont été sélectionnés par quotas selon leur lieu de résidence. En somme nous avons interrogé 1884 personnes (dont 1833 individus sont âgés de plus de 5 ans) sur leurs déplacements d'une journée (mardi, mercredi, jeudi ou vendredi). Nous avons élaboré de multiples visites sur le terrain afin de nous familiariser avec le site. Nous nous sommes présenté à la commune, à la délégation et au lycée d'El Mornaguia, à travers lesquels nous avons fait la connaissance de plusieurs personnes. Ces dernières nous ont introduit auprès de leurs proches, leurs voisins et leurs amis ce qui a facilité énormément notre travail de terrain. Le critère de sélection des ménages enquêtés est celui du «lieu de résidence " vu que l'éloignement des stations de bus est un facteur déterminant dans le choix du moyen de transport collectif à emprunter.

Les déplacements de ces groupes de population vulnérables en matière de mobilité, sont présentés à partir de données d'enquête quantitatives et d'extraits d'entretiens qui mettent en évidence les dimensions sexuée et générationnelle. La connaissance des mobilités de ces groupes de population, de leur rapport aux espaces de vie implique dans un premier temps de prendre en compte l'évolution des pratiques de déplacement à l'échelle de la vie domestique, notamment en matière de chaînes de déplacement ou de pérégrination (Wiel, 1993).

\section{Mobilité quotidienne inégale et contrastée : pratiques différenciées selon le lieu de résidence et les ressources des ménages}

17 Éloignée de $17 \mathrm{~km}$ de la capitale Tunis, l'aire périurbaine d'El Mornaguia s'étend sur 23500 ha dont une grande surface est réservée à l'agriculture. Elle est composée d'une commune centre - El Mornaguia - et de localités périphériques: Sidi Ali Hattab, Hmaiem, Bouregba, El Fejja et El Bassatine. 
Les mobilités dans les aires périurbaines du Grand Tunis. Étude de cas d'El M...

5

Figure 1. Situation d'El Mornaguia dans le Grand Tunis, 2019

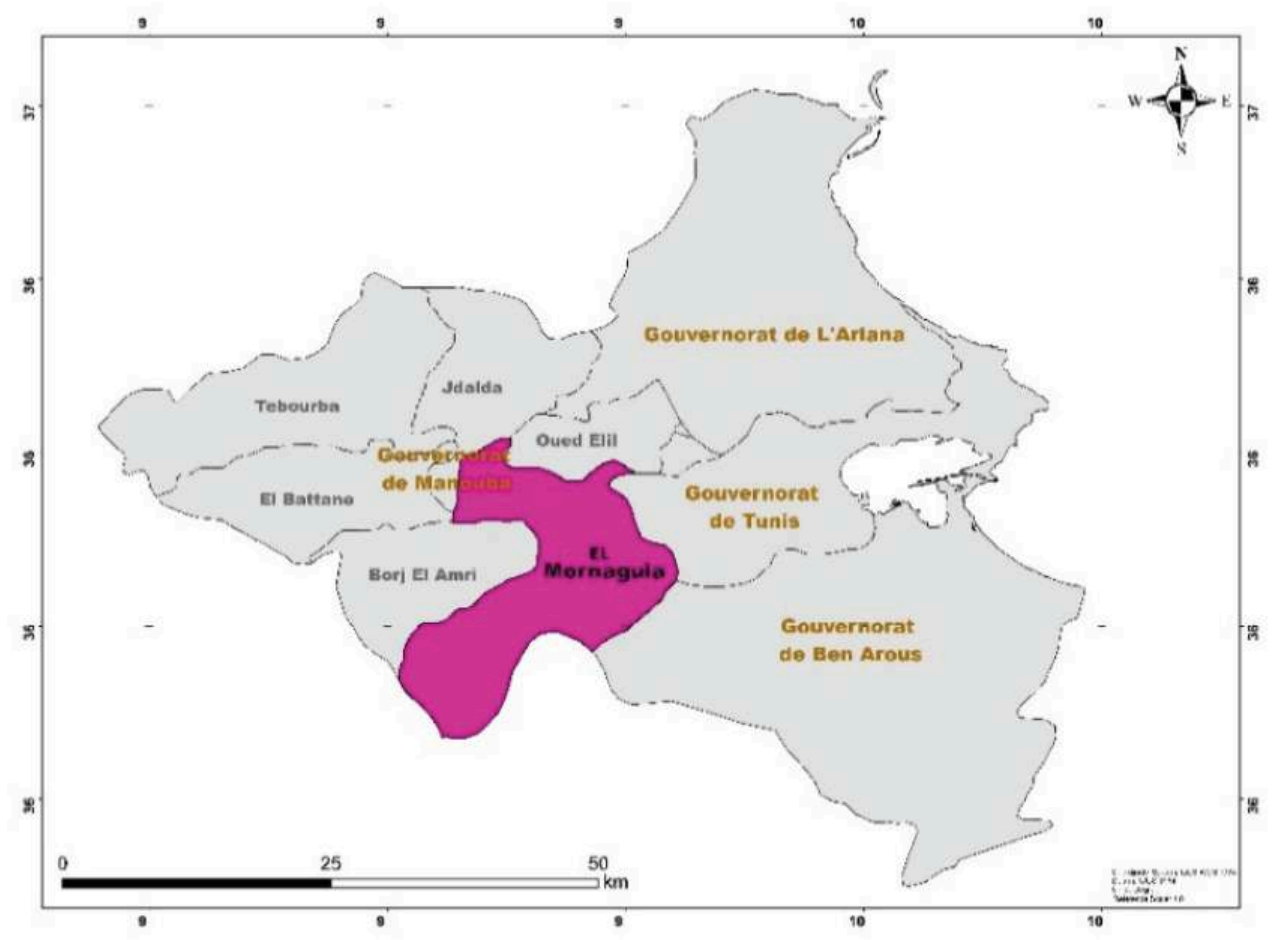

Figure 2. Découpage administratif d'El Mornaguia, 2019

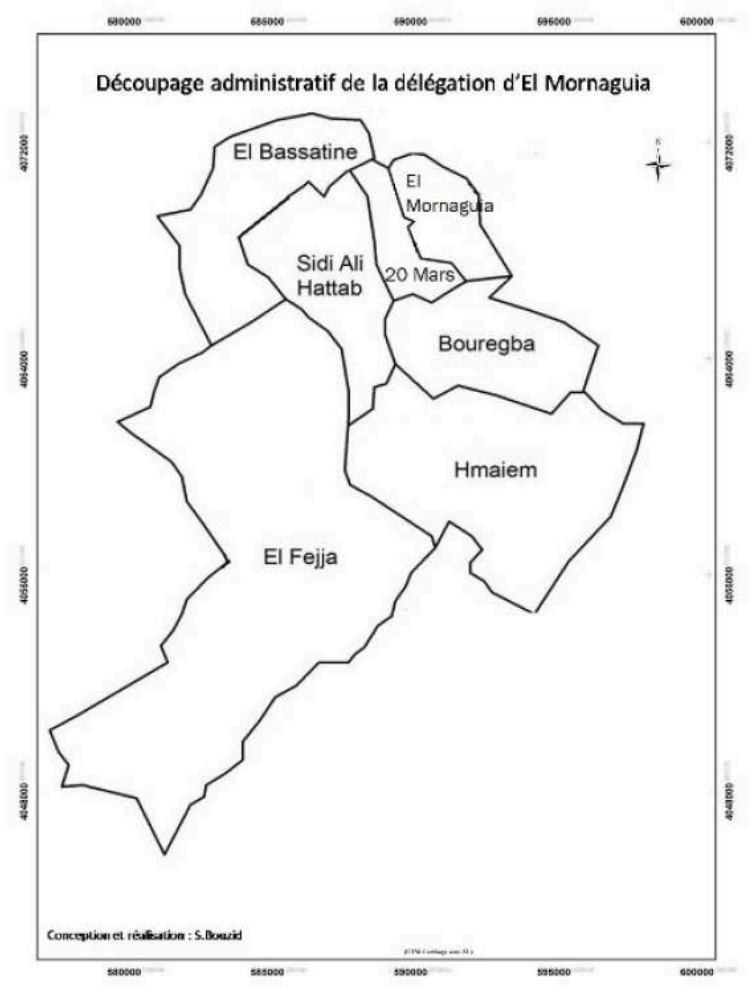

Populations vulnérables, 6 | 2020 


\section{1) Mobilité différenciée en fonction du lieu de résidence}

La mobilité se mesure en nombre de déplacements par jour et par personne ( 5 ans et plus). Ce nombre est obtenu en rapportant le nombre total des déplacements effectués par les personnes enquêtées la veille de l'enquête à la population des individus de 5 ans et plus : la mobilité dans l'aire périurbaine d'El Mornaguia est de 2,21 déplacements par personne et par jour.

Le tableau suivant nous donne un aperçu de la mobilité par lieu de résidence dans l'aire périurbaine d'El Mornaguia :

Tableau 1. Mobilité selon le lieu de résidence

\begin{tabular}{|l|l|l|l|l|l|}
\hline \multicolumn{2}{|l|}{ Milieu } & $\begin{array}{l}\text { Nombre de } \\
\text { ménages } \\
\text { enquêtés }\end{array}$ & $\begin{array}{l}\text { Nombre } \\
\text { d'individus }\end{array}$ & $\begin{array}{l}\text { Nombre } \\
\text { d'individusde 5 ans } \\
\text { et plus }\end{array}$ & Mobilité \\
\hline $\begin{array}{l}\text { Milieu } \\
\text { communal }\end{array}$ & $\begin{array}{l}\text { El } \\
\text { Mornaguia }\end{array}$ & 163 & 741 & 731 & 2,42 \\
\hline \multirow{4}{*}{$\begin{array}{l}\text { Localités } \\
\text { périphériques }\end{array}$} & $\begin{array}{l}\text { Sidi Ali } \\
\text { Hattab }\end{array}$ & 28 & 124 & 108 & 2,29 \\
\cline { 2 - 7 } & Houregba & 35 & 184 & 177 & 2,29 \\
\cline { 2 - 7 } & El Fejja & 43 & 167 & 161 & 2,09 \\
\cline { 2 - 7 } & El Bassatine & 57 & 210 & 205 & 2,98 \\
\hline \multirow{2}{*}{ Total } & 362 & 458 & 451 & 2,21 \\
\hline
\end{tabular}

Source : Bouzid S., enquête sur la mobilité d'El Mornaguia (2014)

Les niveaux de mobilité sont relativement homogènes entre les différentes localités de résidence, allant de 2,42 à 1,98 . Le milieu communal ${ }^{1}$ se distingue par son niveau de mobilité légèrement supérieur à celui des autres localités périphériques. Ceci s'explique par le contexte urbain augmentant les possibilités d'activités (loisirs, équipements socio-collectifs, achats, etc.) et l'offre multiple des moyens de transports collectifs (concurrence des moyens de transport collectif).

21 Les habitants des localités périphériques de l'aire périurbaine d'El Mornaguia se caractérisent par des distances et des durées de déplacements plus longues et par la forte utilisation des transports collectifs par rapport au milieu communal. Quant aux distances parcourues par les chefs des ménages actifs du périurbain périphérique d'El Mornaguia $(30,26 \mathrm{~km})$, elles sont plus longues que celles du milieu communal $(28,3 \mathrm{~km})$. Ceci est expliqué par les distances plus longues que doivent traverser les périurbains des périphéries pour se rendre sur leur lieu de travail.

Tableau 2. Motifs des déplacements à El Mornaguia (avant $10 \mathrm{~h} \mathrm{du}$ matin) 


\begin{tabular}{|l|l|l|}
\hline & Nombre de déplacements & $\%$ \\
\hline Domicile => travail & 1074 & 33,6 \\
\hline Domicile => études & 1136 & 35,6 \\
\hline Domicile => autres & 989 & 30,8 \\
\hline Total & 3199 & 100 \\
\hline
\end{tabular}

Source : Bouzid S., enquête sur la mobilité d'El Mornaguia (2014)

En examinant les lieux de travail des chefs des ménages actifs, nous constatons que :

- L'attraction du centre-ville Tunis est nette. Il assure l'emploi de 31,3\% des chefs de ménages actifs. Ceci témoigne du rôle attractif de la capitale en tant que métropole et confirme le rôle de l'infrastructure routière dans le développement des migrations alternantes.

- Le gouvernorat de La Manouba attire $24,7 \%$ des chefs de ménages actifs.

- La commune d'El Mornaguia assure l'emploi de 10,2\% de l'ensemble des chefs de ménages actifs contre $7,8 \%$ dans les secteurs périphériques.

- Le reste du Grand Tunis attire 9,5\% des chefs de ménages actifs.

Malgré un choix résidentiel périphérique, les actifs ont des pratiques de mobilités étendues et ils revendiquent une forte appétence citadine. Pour lors, nous pouvons

affirmer que

l'aire périurbaine d'El Mornaguia est loin d'offrir l'anonymat et l'isolement du centreville Tunis.

\section{2) Mobilité différenciée selon la position dans le cycle de vie}

L'effet de l'âge intervient au début et à la fin du cycle de vie. C'est aux deux extrémités du cycle de vie qu'on rencontre les mobilités les plus extrêmes (jeunes) et les plus faibles (aînés) comme le montre le graphique suivant : 
Figure 3. Mobilité par classes d'âges à El Mornaguia

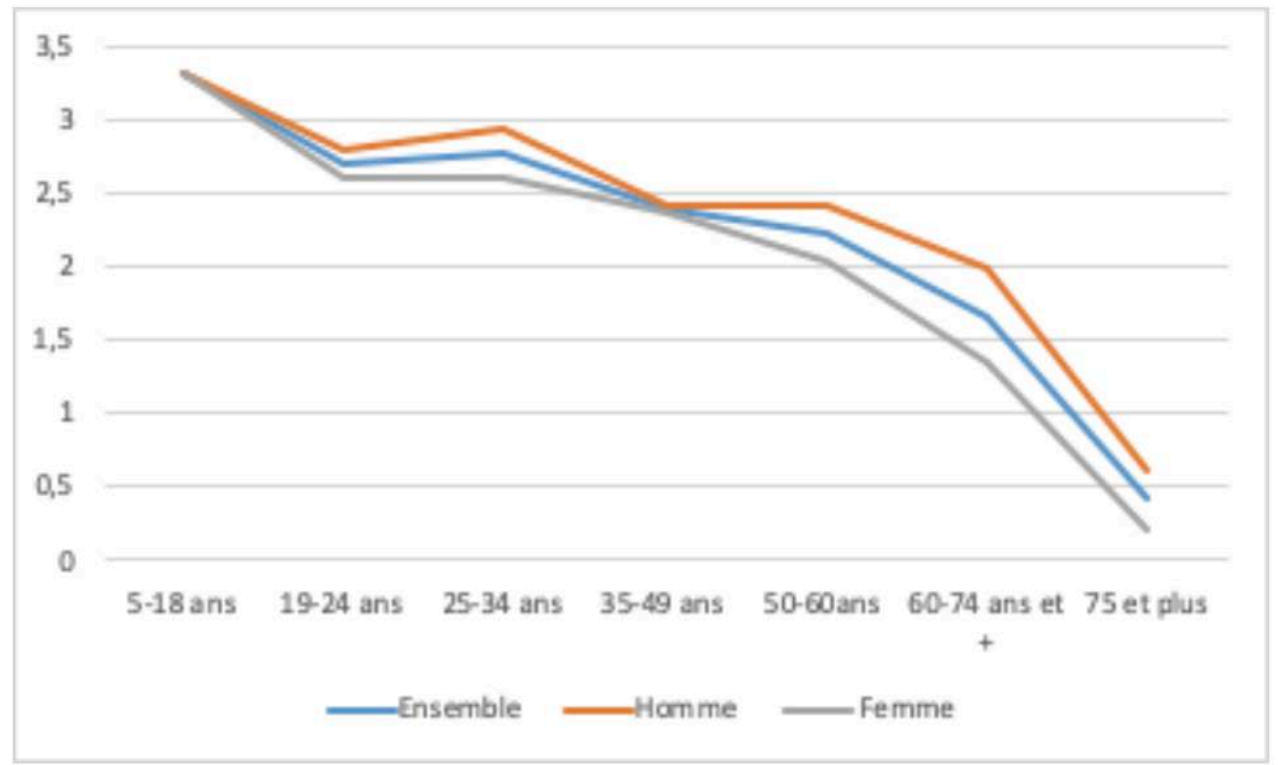

Source : Bouzid S., enquête sur la mobilité d'El Mornaguia (2014)

En poussant encore nos analyses et en examinant le nombre des déplacements selon la position dans le cycle de vie par jour de semaine, nous constatons que :

- Les jeunes (5-18 ans) se caractérisent par la mobilité la plus élevée (3,31). Ceci s'explique tout d'abord par les déplacements contraints (études) qu'effectuent ces jeunes quotidiennement pour se rendre dans les établissements scolaires. L'aire périurbaine d'El Mornaguia étant équipée d'écoles primaires, les déplacements des écoliers peuvent être effectués à pied. Seules la localité d'El Fejja et la commune d'El Mornaguia sont équipées de collèges, les jeunes d'El Fejja et d'El Mornaguia pouvant donc se déplacer à pied vers le collège. Les jeunes des autres localités (Sidi Ali Hattab, Hmaiem, El Bassatine, Bouregba) effectuent des déplacements mécanisés à cet effet. Seule la commune d'El Mornaguia est équipée d'un lycée, cet établissement accueille ainsi tous les lycéens de l'ensemble de l'aire périurbaine. Seuls les lycéens qui résident dans le milieu communal peuvent arriver au lycée à pied, mais les jeunes des autres localités effectuent des déplacements mécanisés pour s'y rendre.

- Les actifs (19-60 ans) se caractérisent par un nombre moyen de déplacements élevé $(2,52)$.

- Les aînés se caractérisent par la mobilité la plus faible (60-74 ans et + avec une mobilité de $1,66)$.

Ainsi, nous pouvons affirmer que le nombre de déplacements à El Mornaguia est tributaire de la position dans le cycle de vie de l'individu.

\section{3) Les hommes se déplacent plus que les femmes}

En matière de mobilité, il existe de fortes disparités entre les individus de sexe différent. À El Mornaguia, si nous prenons en compte la mobilité totale tous modes confondus, nous constatons que les hommes réalisent en moyenne 2,62 déplacements quotidiens par personne contre 1,80 pour les femmes. 
Tableau 3. Nombre moyen de déplacement par jour et par personne selon le sexe

\begin{tabular}{|l|l|l|l|}
\hline & Tous modes & MAP & Modes motorisés \\
\hline Mobilité totale & 2,21 & 1,05 & 1,21 \\
\hline Hommes & 2,62 & 0,9 & 1,72 \\
\hline Femmes & 1,80 & 1,2 & 0,6 \\
\hline
\end{tabular}

$$
\text { À l'îgén }
$$
intervient au début et à la fin du cycle de vie. C'est aux deux extrémités du cycle de vie qu'on rencontre les mobilités les plus extrêmes (jeunes et aînés), l'âge induit ainsi des problématiques spécifiques. Les adolescents, particulièrement démunis en termes d'autonomie spatiale, présentent généralement une mobilité locale, centrée sur le lieu de résidence. Pour les aînés, avec l'abaissement des capacités physiques, l'espace de vie se rétracte, par seuil, jusqu'au repli complet sur l'espace du logement. La question de l'accès aux services fondamentaux, notamment aux soins, et l'accompagnement dans les déplacements, constituent alors un enjeu considérable. Les déplacements des femmes seules (chefs de leurs ménages) sont aussi problématiques en périurbain. Les déplacements de ces groupes de population en difficulté de mobilité, sont présentés à partir de données d'enquête quantitative et d'extraits d'entretiens qui mettent en évidence les dimensions sexuée et générationnelle. 


\section{1) Jeunes périurbains d'El Mornaguia : refus du mode de vie périurbain}

Dans les espaces périurbains dilués, les transports collectifs s'avèrent vite contraignants en matière de fréquences et d'horaires. Dans un mode périurbain où il n'y a pas de multiples choix de transport, l'autonomie de mobilité des jeunes pose problème, particulièrement celle des adolescents qui sont en phase d'acquisition de l'autonomie spatiale (Thomann, 2009). Cette autonomie va se traduire par la recherche d'une inscription spatiale qui a un caractère structurant en matière d'identité et d'inscription spatiale.

Ainsi, les jeunes (5-18 ans) résidant à El Mornaguia ont leurs pratiques propres de mobilité pour accéder à leurs établissements scolaires, soit l'école, soit le collège, soit le lycée. Selon la localité de résidence et ses équipements éducatifs, la mobilité ainsi que les distances parcourues pour les jeunes sont comme suit :

Figure 4. Nombre de déplacements et distance parcourue des jeunes

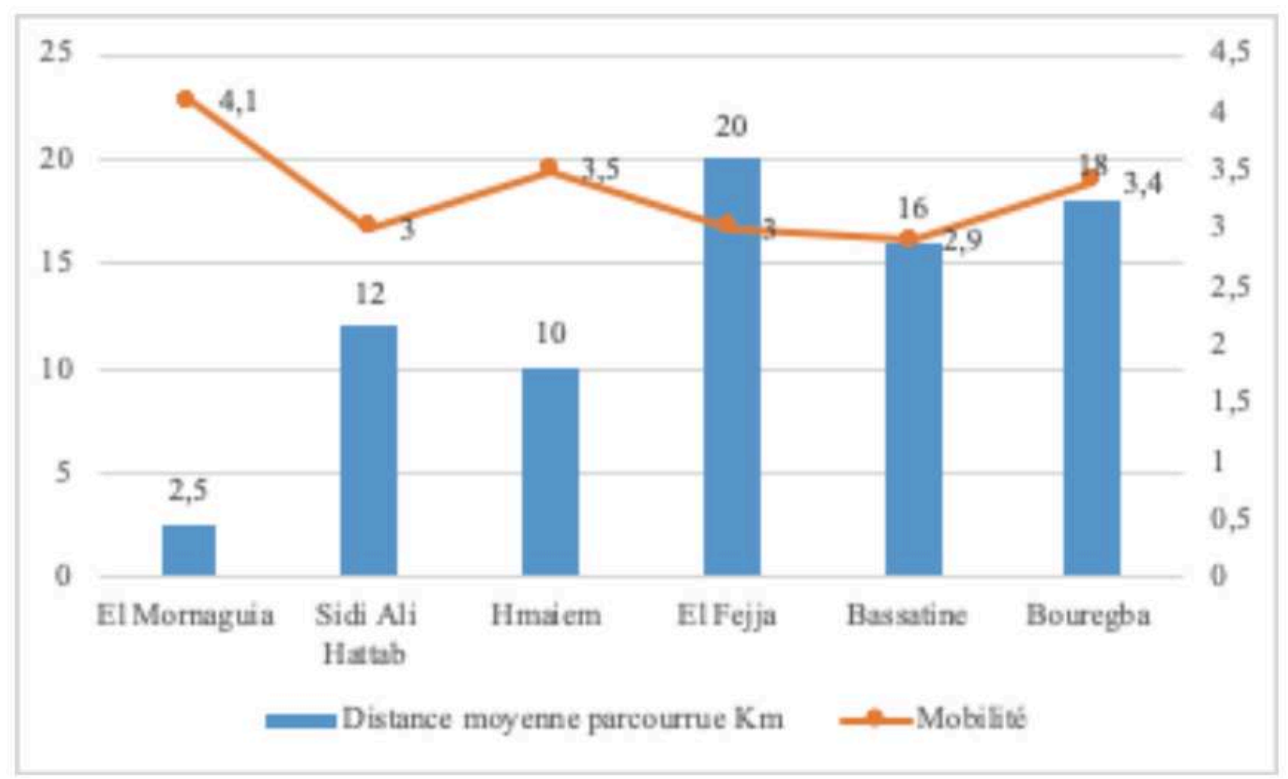

Source : Bouzid S., enquête sur la mobilité d'El Mornaguia (2014)

\section{a) Les déplacements scolaires des jeunes}

\section{Les déplacements vers l'école}

Toutes les localités de l'aire périurbaine d'El Mornaguia sont équipées d'écoles primaires. Tous les déplacements vers l'école peuvent être effectués à pied vu les morphologies spatiales de toutes les localités, adéquates à la marche à pied.

Ainsi, $98 \%$ des déplacements vers les écoles primaires sont effectués à pied contre $2 \%$ pour ceux qui sont accompagnés par leurs parents en voiture particulière, qui les déposent avant d'aller à leur travail. Les déplacements vers l'école permettent aux enfants d'acquérir leur autonomie spatiale. Les déplacements vers les écoles primaires ne posent donc pas de problèmes dans l'aire périurbaine d'El Mornaguia, néanmoins le problème se pose pour les noyaux d'habitat non réglementaire 
éloignés des centres des localités. Les enfants de ces localités doivent se déplacer sur des distances longues et non sécurisées pour pouvoir arriver à l'école.

\section{Les déplacements vers le collège}

Le tissu urbain de la commune d'El Mornaguia permet aux jeunes d'acquérir une certaine autonomie de déplacement: $91 \%$ des collégiens résidant à El Mornaguia viennent à pied. Bien que la commune s'étale sur $2,1 \mathrm{~km}$ de long et 1,3 km de large, ce qui est praticable à pied, on constate que $9 \%$ des collégiens viennent accompagnés en voiture particulière par leurs parents.

Dès l'âge de 11-12 ans, les jeunes des localités périphériques démunies de collège commencent à se déplacer seuls pour aller au collège et commencent à acquérir leur autonomie grâce au transport en commun utilisé (transport scolaire ou transport rural dans notre cas). Les résultats de notre enquête reflètent bien l'importance du transport collectif (bus), adapté aux horaires des collèges d'El Mornaguia, dans la desserte des équipements scolaires.

Figure 5. Collégiens en heure creuse devant le collège Abou Kacem Chabbi-El Mornaguia

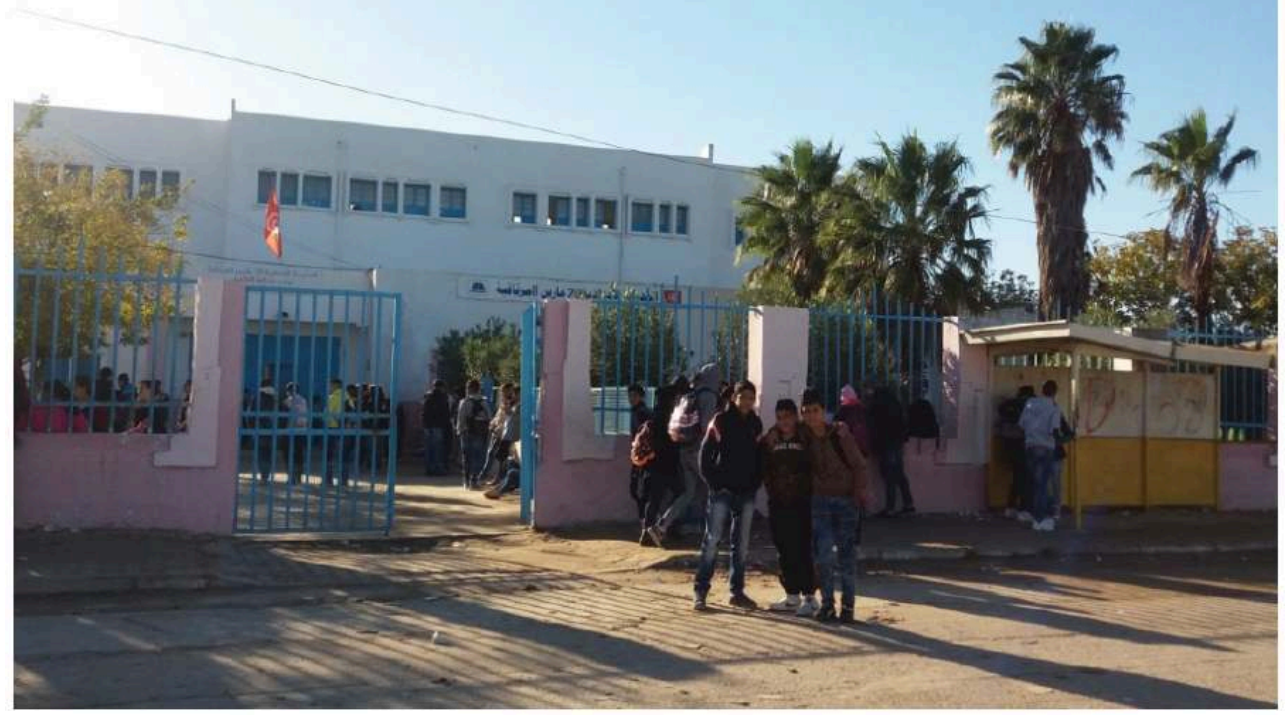

Cliché : S.Bouzid (mars 2014) 
Figure 6. Moyens de transport utilisé par les collégiens selon le lieu de résidence

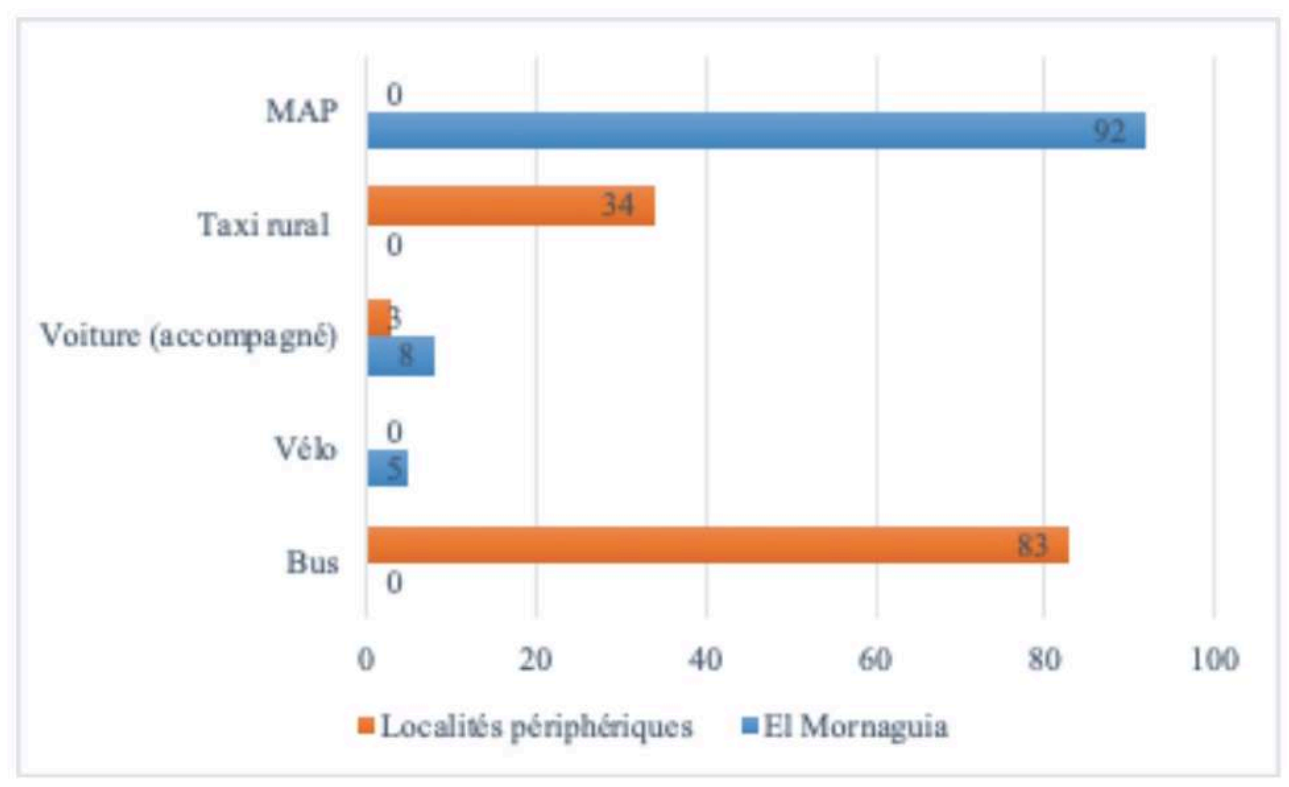

Source : Bouzid S., enquête sur la mobilité d'El Mornaguia (2014)

\section{Les déplacements vers le lycée}

Avec l'âge, les adolescents changent d'établissement scolaire, du collège au lycée, et vont s'approprier à cette occasion de nouveaux modes de transport. De plus, l'entrée au lycée marque une nouvelle étape, avec la possibilité accordée de sortir de l'enceinte scolaire avant l'heure du car et la découverte de la ville d'El Mornaguia. Avec ce changement d'établissement, il est certain que cela favorise une certaine ouverture citadine. Des amitiés se nouent entre adolescents d'El Mornaguia et adolescents de ses périphéries. Les commerces, les cafés, les restaurants, la maison des jeunes, la maison de la culture, la bibliothèque publique, etc., peuvent devenir des supports de loisir et de sociabilité des lycéens. L'espace des loisirs et des sociabilités s'élargit donc, alors que les liens avec le quartier de résidence se distendent. La question de la mobilité pour les loisirs et les sociabilités se pose donc rapidement avec acuité et est souvent un facteur premier de conflit dans le ménage. Ce sont donc les adolescents du périurbain périphérique qui souffrent le plus de l'éloignement de la ville et de ses commodités :

En tant que captive du transport collectif, je ne suis pas du tout satisfaite de l' offre de transport en commun et particulièrement du transport scolaire. Le bus de transport scolaire vie nt toujours en retard, ce qui engendre pour moi des problèmes avec l' administration du lycée. Ainsi pour le billet, je serai obligée de ramener l'un de mes parents pour obtenir le billet. Si je rate le bus scolaire, je prends le bus $n^{\circ} 23$ $\mathrm{D}$ il me faut faire un voyage pénible pour arriver au lycée tout en sachant que la durée du trajet entre Tbaltich et El Mornaguia est de 30 minutes par bus. Le bus de transport scolaire vient normalement à $7 \mathrm{~h}$ du matin pour pouvoir ramener les

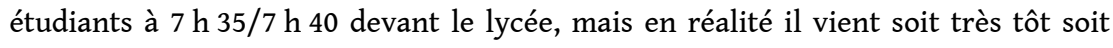
très tard... (Nermine El Balrouthi, Sidi Ali Hattab, 17 ans, 2016)

L'utilisation des transports collectifs par les adolescents est ambivalente : d'une part c'est un temps contraignant, il fixe leurs horaires de déplacement en fonction des horaires des bus scolaires et d'autre part il influence leur socialisation. Ainsi le temps 
de déplacement est important pour les jeunes et dans la même optique Kaufmann et Flamm affirment que "L'émancipation de l'enfant puis de l'adolescent se construit autour de l'autonomie de déplacement » (Kaufmann et Flamm, 2002, p. 3).

Pour ces adolescents, le fait de résider dans l'espace périurbain entraîne une distorsion forte entre pratiques rêvées et pratiques spatiales réelles. Les difficultés de mobilité conduisent à une forte envie d'aller ailleurs, très souvent traduite en envie de ville avec ses commodités. On peut d'ailleurs se demander si les espaces périurbains ne fabriquent pas des urbains (expérience dans le transport collectif, transmission des expériences familiales, etc.).

résument le processus d'autonomisation chez les jeunes comme suit : « Il commence par l'apprentissage de la marche, se poursuit avec l'autorisation d'aller jouer tout seul chez des copains, puis de se déplacer seul en journée, puis le soir, chaque fois avec négociation [...], négociation dont les termes sont différenciés entre garçons et filles »

(Kaufmann et Flamm, 2002, p. 17). Ainsi, l'indépendance spatiale augmente fortement avec l'âge de l'adolescent. En outre la fille est toujours plus contrôlée et plus surveillée que le garçon.

\section{b) Facteurs influençant la mobilité des jeunes à El Mornaguia}

\section{Lieu de résidence : manque d'urbanité du périurbain périphérique}

Le premier facteur qui influence la mobilité des jeunes est la localisation résidentielle. L'installation en périurbain d'El Mornaguia influence fortement les programmes d'activités des ménages et par conséquent la mobilité quotidienne des jeunes. Ce type d'installation en périurbain d'El Mornaguia implique des contraintes et oriente les pratiques d'habitation et de déplacement. La commune offre des équipements éducatifs et extrascolaires tandis que les périphéries d'El Mornaguia ne les offrent pas. Nous avons constaté que $69 \%$ des jeunes qui effectuent des activités extrascolaires habitent le milieu communal et que $83 \%$ des lycéens qui effectuent des déplacements - hors sphère études - résident dans le milieu communal contre $21 \%$ pour les résidents $d u$ périurbain périphérique.

\section{Situation économique des ménages}

$\mathrm{Au}$ cours de notre analyse, nous nous sommes également préoccupé de la situation économique des ménages (pouvoir d'achat) qui a sans doute un effet sur la mobilité des jeunes et par la suite sur leur socialisation. Ainsi, les jeunes issus des ménages disposant de voitures particulières se déplacent sur des distances plus longues que ceux qui n'en disposent pas ( $25 \mathrm{~km}$ contre $9 \mathrm{~km}$ en moyenne). Les jeunes dont les parents sont des cadres supérieurs et des cadres moyens se déplacent en dehors de leur lieu de résidence durant les week-ends.

Nous avons ainsi montré que les jeunes issus des ménages aisés disposaient d'une bonne maîtrise du champ spatial et qu'ils s'affranchissaient très largement des barrières géographiques. Cela tient bien entendu au différentiel observé quant à l'accès au mode automobile, mais également à l'éventail de choix dont ils disposent pour définir leurs lieux d'achats, de loisirs, etc. Les plus favorisés ont en effet une plus 
grande ouverture sur les ressources non locales (amitiés distantes, éloignement familial, réseaux sociaux fournis et dispersés).

En revanche, l'insertion spatiale des jeunes issus des ménages modestes est davantage basée sur la proximité. Les individus les plus aisés développent des identités territoriales multiples et complexes tandis que celles des plus démunis se caractérisent par un ancrage résidentiel fort et par des liens faibles avec les espaces lointains.

Nous pouvons donc affirmer que selon le milieu socio-économique des parents, ces différences entre les adolescents peuvent être grandes : nous constatons que les plus vulnérables subissent souvent des temps de transport longs et pénibles et ont une expérience de la ville réduite voire inexistante. Selon les résultats d'une enquête effectuée auprès des jeunes périurbains (13-18 ans) sur le territoire de la Dombes (France), Marie Goyon affirme que «selon le milieu socio-économique des parents, ces différences entre les adolescents peuvent être grandes : nous constatons que les plus défavorisés subissent souvent des temps de transport longs et pénibles, ont une expérience de la ville réduite voire inexistante » (Goyon , 2009).

\section{2) Mobilités et ancrage des aînés dans l'aire périurbaine d'El Mornaguia}

Les périurbains âgés ne se déplacent pas au hasard. Leur mobilité est calculée puisqu'il s'agit d'une catégorie générationnelle fragile dont le mode d'habiter et le rapport au lieu de résidence sont en étroite relation avec leurs capacités physiques. Parmi les aînés, il y a une "variabilité des pratiques de mobilité quotidienne» (Lord, Joerin et Thériault, 2009). Les aînés ne forment donc pas un groupe socio-économiquement homogène, cela s'exprime notamment dans leurs pratiques spatiales (Lord, Joerin et Thériault, 2009). Leurs déplacements peuvent augmenter lors du passage à la retraite. $\mathrm{C}$ ertains individus âgés peuvent se déplacer autant, voire plus que les cohortes de travailleurs plus jeunes. Ainsi, l'âge n'est pas l'unique déterminant de la mobilité quotidienne dans la vieillesse, il doit être considéré en relation avec d'autres dimensions qui y sont étroitement liées, notamment l'autonomie fonctionnelle. En fait, seul un âge relativement avancé, souvent accompagné de limitations d'autonomie et de problèmes de santé, réduit de manière drastique le nombre de déplacements des individus.

51 Les aînés d'El Mornaguia représentent $21 \%$ de l'ensemble des individus $(\mathrm{n}=24)$. Ces aînés défendent âprement les avantages de leur lieu d'habitation et 87 \% d'entre eux sont très satisfaits de leur lieu de résidence : tous les aînés du milieu communal sont très satisfaits tandis que ceux des périphéries réclament la nécessité de renforcer l'offre de transport collectif et la satisfaction de quelques besoins en équipements et services afin de réduire leurs déplacements quotidiens.

Sur l'ensemble des aînés de l'aire périurbaine d'El Mornaguia et en comparant leurs déplacements avec la moyenne de l'ensemble des individus de cette aire, nous remarquons une légère baisse du nombre des déplacements des aînés de 60-74 ans et une baisse importante pour les aînés de 75 ans et plus. Les aînés de 60-74 ans ont des niveaux de mobilité plus faibles que la moyenne (1,66 déplacements par jour). Ainsi les ainés, ayant plus de 75 ans, ne représentent que 8 personnes et ils sont caractérisés par une mobilité plus réduite ( 0,42 déplacement par jour). 
53 En analysant le nombre des déplacements par lieu de résidence, nous constatons que les aînés résidant dans le milieu communal se caractérisent par une légère augmentation du nombre de déplacements par rapport aux aînés des localités périphériques $(2,03$ déplacements contre 1,59$)$. Ceci peut être expliqué par les caractéristiques urbaines du lieu de résidence puisque le milieu communal se caractérise par la multiplicité des lieux de rencontre (les cafés, les lieux de culte, les associations, les bureaux des partis politiques, les équipements, les services, etc.), contre uniquement les cafés et une mosquée pour les localités périphériques. Si nous poussons encore la réflexion, nous notons que les hommes se déplacent plus que les femmes. Ces dernières ont 1,68 déplacements contre 0,99 pour

les hommes

L'analyse fine des pratiques des mobilités des aînés révèle une typologie à trois termes pour ces ainés : les casaniers, les aînés avec une mobilité locale et les aînés avec une mobilité à structure éclatée. Leur répartition se présente comme suit :

Figure 7. Classification des mobilités des aînés

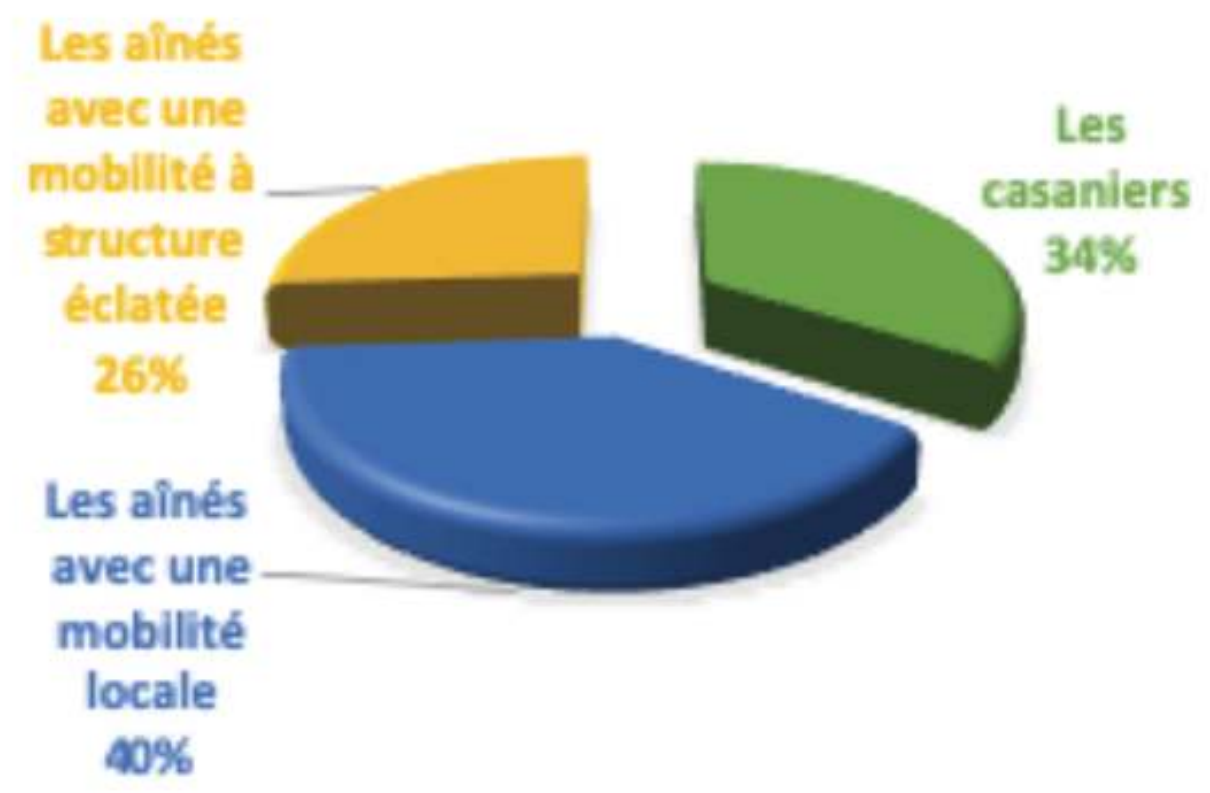

Source : Bouzid S., enquête sur la mobilité d'El Mornaguia (2014)

\section{a) Les casaniers ou les assignés territoriaux}

Les casaniers forment une catégorie d'aînés qui ne disposent pas de voiture particulière dans notre cas. L'aire de déplacement fréquentée de manière autonome (de 0 à $1 \mathrm{~km}$ ) est représentée par la localité de résidence, tout en pouvant être limitée au domicile dans certains cas. Ces casaniers se caractérisent par un immobilisme autour du lieu de résidence. Ce type d'aînés représente dans notre enquête $8,3 \%(n=2)$ de l'ensemble des aînés enquêtés. Ces aînés casaniers ont plus de 75 ans. 


\section{b) Aînés avec une mobilité locale}

57 Les aînés avec une mobilité locale vivent en couple et/ou avec leurs enfants. Ils représentent la typologie la plus dominante à raison de $75 \%(\mathrm{n}=18)$ de l'ensemble des aînés d'El Mornaguia (5 résident aux périphéries et 9 au milieu communal). L'aire géographique de déplacement calculée est ici comprise entre 3 et $9 \mathrm{~km}$, ce qui signifie des déplacements courts se cantonnant le plus souvent aux lieux strictement voisins à leur localité de résidence. À ce stade de la réflexion, nous qualifions cette mobilité de concentrée et de calculée. Les aînés avec une mobilité locale sont les plus attachés aux commerces de l'aire périurbaine d'El Mornaguia:

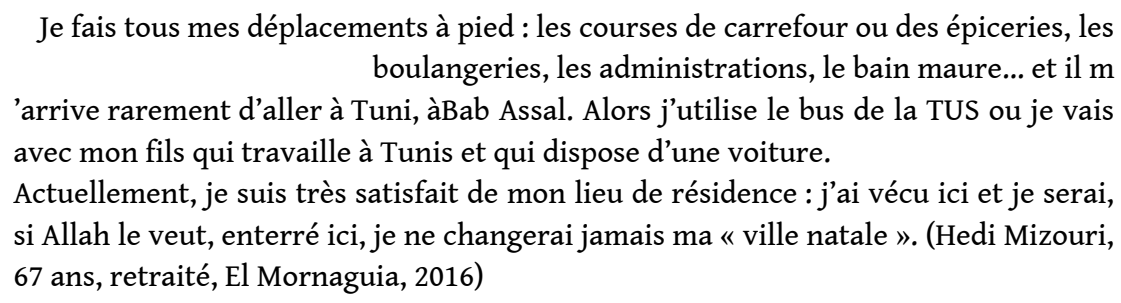

\section{c) Aînés avec une mobilité à structure éclatée}

Les aînés avec une mobilité à structure éclatée se distinguent des aînés avec une mobilité locale par l'étendue de leurs déplacements, avec une aire géographique étendue pouvant atteindre $20 \mathrm{~km}$. Ces aînés ont une mobilité à structure éclatée, similaire à celle observée auprès des ménages actifs. Si les "mobiles à structure éclatée » sont si mobiles, c'est en partie parce qu'ils ont la particularité de vivre en famille et d'être en relative bonne santé. Ils ne représentent que $26 \%$ de l'ensemble des aînés d'El Mornaguia $(\mathrm{n}=6)$. Leurs lieux fréquentés sont essentiellement le centre-ville Tunis ou le gouvernorat de la Manouba.

Pour conclure, nous notons que la réduction de la mobilité des personnes âgées induit une appropriation plus forte de l'environnement résidentiel et une meilleure insertion dans les réseaux sociaux de proximité qui profitent à tout le voisinage (commerces, partis politiques, lieux de culte, visite familiale, etc.). Nous assistons à un réinvestissement dans la localité de résidence qui révèle un apprentissage de la vie locale - voire villageoise (pour aînés avec une mobilité locale et pour ceux avec une mobilité à structure éclatée). Les aînés sont souvent des résidents présents. Du fait de leur mobilité limitée, ils surinvestissent l'aire périurbaine d'El Mornaguia.

\section{3) Femmes seules en périurbain d'El Mornaguia : entre déplacements quotidiens et gestion de la vie duménage}

Que signifie être une femme dans le périurbain tunisois aujourd'hui? La situation de chacune dépend de son accès au travail, de ses horaires, de la localisation de son lieu de travail et bien évidemment de son contexte familial. Les espaces périurbains, par la multiplication des déplacements qu'ils imposent, peuvent paraître comme inadaptés à l'articulation entre travail et vie familiale. « Les femmes continuent à y porter la charge mentale de l'éducation des enfants et sont donc soumises à de nombreuses tensions pour organiser la vie domestique " (Thomann, 2009 ; Pinson et Thomann, 2001). Alors comment s'organisent leurs mobilités quotidiennes, en particulier celles des femmes seules responsables de leur ménage (les femmes veuves ou divorcées)? 
61 Dans la plupart des sociétés, les hommes se déplacent plus que les femmes. Nous avons déjà montré précédemment que les femmes se déplacent moins que les hommes. Ce constat est dû au fait que ce sont généralement les femmes qui effectuent les activités ménagères à domicile. Cet écart varie selon le pays en question (en fonction des normes sociales). Leur mobilité est faiblement étudiée dans les pays du Sud et surtout pour les femmes seules. Cette dimension par le sexe vient ici étayer le point de vue développé dans cette thèse de pointer les inégalités en matière de mobilité en périurbain d'El Mornaguia.

62 Les femmes interrogées font partie de la classe moyenne, vulnérable et résidant dans le périurbain communal d'El Mornaguia ainsi que ses localités périphériques. Plus des $2 / 3$ des femmes totales interviewées sont des femmes au foyer ou ont cumulé de petits emplois sporadiques ou mal rémunérés (femmes de ménage, couturières, etc.). Les femmes chefs de leurs ménages, résidant dans l'aire périurbaine d'El Mornaguia, représentent 4,2\% (n=15) de l'ensemble des chefs des ménages.

Les femmes chefs de leurs ménages du milieu communal sont toutes satisfaites (100\%) de leurs lieux de résidence, tandis que celles des localités périphériques ne le sont pas et réclament des besoins en équipements et en services. Elles regrettent l'éloignement des commodités qu'offre le milieu communal.

Les distances parcourues par les femmes seules sont de l'ordre de 5,3 km par jour $(1,3 \mathrm{~km}$ pour le milieu communal et $4 \mathrm{~km}$ pour les périphéries). Elles sont alors à mobilité locale dans leur aire de résidence. Si nous comparons cette distance moyenne avec la moyenne enregistrée pour l'ensemble des individus, nous constatons une nette diminution $(5,3 \mathrm{~km}$ contre $17 \mathrm{~km})$. Il est à noter qu'aucune femme seule ne possède le permis de conduire.

Seule une femme s'est déplacée en dehors de l'aire périurbaine d'El Mornaguia, les autres sont restées dans leur localité de résidence. Les motifs de leurs déplacements sont les suivant : $67 \%$ achats, $21 \%$ visites familiales, $12 \%$ autres.

Les femmes seules sont impliquées dans leur lieu de résidence puisque tous leurs déplacements sont effectués dans l'aire périurbaine d'El Mornaguia. Les femmes ne se déplacent pas sur de longues distances. Elles subviennent à tous leurs besoins dans l'aire de résidence. En outre, nous signalons que toutes les femmes ont effectué des déplacements de proximité, c'est-à-dire qu'il n'y a pas de femmes "casanières ». Le périurbain d'El Mornaguia offre un cadre de vie pour renforcer la sociabilité de ses résidentes et ne provoque pas d'isolement résidentiel. Les femmes en périurbain d'El Mornaguia ont davantage de temps pour apprivoiser leur environnement, et ainsi créer des liens de proximité.

Les femmes périurbaines d'El Mornaguia sous-estiment par ailleurs les effets de leur choix modal sur leurs modes de vie. Une large majorité d'entre elles évitent le cœur de l'agglomération, en raison des coûts des déplacements et de leur pénibilité :

Honnêtement, j'évite le déplacement ailleurs d'El Mornaguia et, depuis près d' un an et demi, je ne suis pas allée ailleurs. En fait, je me demande pourquoi aller ailleurs puisqu

'on a tout le nécessaire ici. Auparavant, j'avais besoin de me déplacer à laManouba ou au Denden pour des affaires administratives, mais actuellement non. Je me déplace à Tunis très rarement... (Hbiba Khelifi, 58 ans, veuve, El Mornaguia, 2016) 


\section{Conclusion} habitants de l'aire périurbaine d'El Mornaguia: l'âge, le sexe, le niveau socioéconomique du ménage et l'état de santé. Nous affirmons donc que les conditions sociales et territoriales se combinent pour contraindre la mobilité quotidienne et limiter l'accessibilité des individus en périurbain.

73 Avec la communalisation du territoire national, les périmètres des communes sont modifiés. Les communes prendront en considération les avis/choix des habitants dans les nouvelles prises de décision, il sera alors pertinent d'étudier les conséquences de ces nouvelles prises de décision sur les pratiques de mobilité des ménages en situation de vulnérabilité. Les conditions de vie des ménages périurbains en situation de vulnérabilité s’amélioreront-elles?

\section{BIBLIOGRAPHIE}

Belhareth T. (2004), Transport et structuration de l'espace tunisien, Tunis, Publications de la Faculté des Sciences Humaines et Sociales de Tunis, $612 \mathrm{p}$. 
Belhareth T. (1990), « État, espace urbain et transport collectif. Cas des grandes agglomérations tunisiennes ", Revue tunisienne de géographie, $\mathrm{n}^{\circ}$ 18, p. 55-93.

Belhedi A. (1992), L'aménagement de l'espace en Tunisie : production et reproduction de l'espace, Tunis, Publication de la Faculté des sciences humaines et sociales, $267 \mathrm{p}$.

Bouzid S. (2018), La mobilité dans les aires périurbaines du Grand Tunis. Étude de cas d'El Mornaguia, École nationale d'architecture et d'urbanisme de Tunis, thèse de doctorat en urbanisme et aménagement.

Bouzid S. (2010), Mobilité et étalement urbain, mobilité des jeunes périurbains et inégalités sociospatiale, terrain de réflexion : la commune de Rousset, Aix-en-Provence, Institut d'urbanisme et d'aménagement régional, mémoire de Master Erasmus Mundus études urbaines en régions méditerranéennes, $40 \mathrm{p}$.

Caubel D. (2006), Politiques de transports et accès à la ville pour tous? Une méthode d'évaluation appliquée à l'agglomération lyonnaise, thèse de doctorat en sciences économiques, université de Lyon.

Chabbi M. (2012), L'urbain en Tunisie, Processus et projet, Tunis, NIRVANA, 219 p.

Kahloun H. (2013), Les petites villes de la périphérie de Tunis face à la métropolisation, processus d'urbanisation, autonomisation et défis de la gouvernance, Saarbrücken, PAF, 449 p.

Coquart B. (2018), « L'investissement dans la voiture compense la délocalisation des emplois », Les jours [en ligne], $\mathrm{n}^{\circ} 7$, disponible sur http://lesjours.fr/obsessions/route/ep7-interviewbenoit-coquard/, consulté le 12/12/2019.

Fol S. (2009), La mobilité des pauvres. Pratiques d'habitants et politiques publiques, Paris, Belin, $264 \mathrm{p}$.

Froud J., Johal S., Leaver A. et Williams K. (2002), « Not Enough Money: The Resources and Choices of the Motoring Poor », Competition and Change, vol. 6, n 1, p. 95-111.

Goyon M. (2009), «Jeunesses périurbaines en Dombes : mobilité quotidienne, modèles parentaux et socialisation », colloque de l'ASRDLF, $17 \mathrm{p}$.

Jouffe Y., Caubel D., Fol S. et Benjamin M-B. (2015), «Faire face aux inégalités de mobilité », Cybergeo. European Journal of Geography [en ligne], document 708, mis en ligne le 19 janvier 2015, disponible sur https://journals.openedition.org/cybergeo/26697.

Kaufmann V. (2001), « Mobilité et vie quotidienne : synthèse et question de recherche », Centre de prospective et de veille Scientifique, Synthèses et recherches, $\mathrm{n}^{\circ} 48,57 \mathrm{p}$.

Kaufmann V. (2000), « Mobilité quotidienne et dynamiques urbaines », Lausanne, Presses polytechniques et universitaires romandes, $252 \mathrm{p}$.

Kaufmann V. et Flamm M. (2002), «Famille, temps et mobilité. État de l'art et tour d'horizon des innovations », rapport de recherche pour la CNAF et l'Institut La ville en mouvement, $62 \mathrm{p}$.

Lord S., Joerin F. et Theriault M. (2009), « Évolution des pratiques de mobilité dans la vieillesse : un suivi longitudinal auprès d'un groupe de banlieusards âgés ", Cybergeo. European Journal of Geography [en ligne], document 444, mis en ligne le 23 mars 2009, disponible sur https:// journals.openedition.org/cybergeo/22090.

Makdessi Y., Vichnevskaia T. et Weber A. (2018),

«Introduction. Émergence et usages du concept de vulnérabilité des personnes âgées », Populations vulnérables, $\mathrm{n}^{\circ} 3$. 
Morinaux V. (dir.) (2010), Les mobilités, Paris, SEDES, 229 p.

Nicolas J.-P., Vanco F. et Verry D. (2012), « Mobilité quotidienne et vulnérabilité des ménages », Revue d'économie régionale et urbaine, $\mathrm{n}^{\circ} 1, \mathrm{p} .19-44$.

Pinson D. et Thomann S. (2001), La maison en ses territoires, de la villa à la ville diffuse, Paris, L'Harmattan. $191 \mathrm{p}$.

Orfeuil J-P. (2010), « La mobilité, nouvelle question sociale ? », Sociologies [en ligne], Dossiers, mis en ligne le 27 décembre 2010, disponible sur http://sociologies.revues.org/3321.

Orfeuil J.-P. (2004), Transports, pauvretés, exclusions, Paris, Éditions de l'Aube, p. 27-47.

Després C., Pinson D. et Ramadier T. (2006), « Étalement urbain et discrimination par l'automobilité : Aînés et jeunes à Aix », in Pinson D. (dir.), Métropoles au Canada et en France. Dynamiques, politiques et cultures, Presses universitaires de Rennes, p. 47-63.

Rhodes Thomann S. (2009), Familles, femmes et générations exposées à l'étalement urbain. Installation résidentielles et dépendance automobile dans l'aire métropolitaine marseillaise, Aix-en-Provence, Institut d'urbanisme et d'aménagement régional, thèse de doctorat en aménagement de l'espace, urbanisme.

Saujot M. (2012), « La mobilité, l'autre vulnérabilité énergétique », Policy Brief, n 5, 6 p.

Troin J-F. (2007), Les métropoles des « Sud», Paris, Ellipses, 158 p.

Wiel M. (1999), La transition urbaine, Liège, Margada, 149 p.

\section{NOTES}

1. Avant 2016, le territoire tunisien était composé d'un milieu communal et d'un milieu rural. Ces deux milieux formaient les 24 gouvernorats du pays. Depuis la communalisation du territoire national (2016), les communes sont au nombre de 350 publié par le ministère des Affaires locales. L'ensemble du territoire tunisien est désormais couvert par des communes, incluses à l'intérieur des 24 gouvernorats.

\section{RÉSUMÉS}

Cet article analyse les conséquences d'une installation en périurbain à El Mornaguia - qui se situe dans la deuxième couronne périurbaine $d u$ Grand Tunis - sur les pratiques de mobilité, à partir d'une enquête par questionnaire auprès de $5 \%$ des ménages (362 ménages) et des entretiens semi-directifs effectués avec des groupes spécifiques. Les conséquences de l'installation en périurbain à El Mornaguia sont étudiées tout d'abord sur l'ensemble des ménages et par la suite sur des groupes spécifiques de population, à savoir les aînés (60 ans et plus), les jeunes (12-18 ans) et les femmes seules. L'enquête montre que la mobilité quotidienne à El Mornaguia reflète des inégalités sociales. Elle est influencée également par l'âge dont l'effet intervient au début et à la fin du cycle de vie. Elle est influencée également par le genre puisque les hommes se déplacent plus que les femmes. 
This article analyses the consequences of a periurban installation in El Mornaguia, which is located in the second suburb of Greater Tunis on mobility practices from a questionnaire survey of $5 \%$ of households ( 362 households) and semi-direct interviews with specific groups. The consequences of urban peri-urban settlement of El Mornaguia are studied first, on all households and then on specific groups of the population, namely the elderly (60 years and over), young people (12-18 years) and women alone. The survey shows that daily mobility in El Mornaguia reflects social inequalities. It is also influenced by age at the beginning and end of the life cycle. It is also influenced by gender as men travel more than women.

\section{INDEX}

Mots-clés : périurbain, mobilité, inégalités socio-spatiales, jeunes, aînés, femmes seules Keywords : suburban, mobility, socio-spatial inequalities, youth, seniors, single women

\section{AUTEUR}

\section{SOUHIR BOUZID}

Docteur en urbanisme et aménagement

École nationale d'architecture et d'urbanisme de Tunis, laboratoire Gouvernance, ambiances et développement des villes 